\title{
THE RÔLE OF CATEGORICAL STRUCTURES IN INFINITESIMAL CALCULUS
}

\author{
William Steingartner ${ }^{1}$, Darko Galinec ${ }^{2}$ \\ ${ }^{1}$ Department of Computers and Informatics, Technical University of Košice \\ Košice, Slovakia \\ ${ }^{2}$ Department of Informatics and Computing, Zagreb Polytechnic for Technical Sciences \\ Zagreb, Croatia \\ ${ }^{I}$ william.steingartner@tuke.sk, ${ }^{2}$ darko.galinec@tvz.hr
}

\begin{abstract}
The development of mathematics stands as one of the most important achievements of humanity, and the development of the calculus, differential calculus and integral calculus is one of the most important achievements in mathematics. Differential calculus is about finding the slope of a tangent to the graph of a function, or equivalently, differential calculus is about finding the rate of change of one quantity with respect to another quantity. On the other hand, integration is an important concept in mathematics and, together with its inverse, differentiation, is one of the two main operations in calculus. Integrals and derivatives became the basic tools of calculus, with numerous applications in science and engineering. The category theory is a mathematical approach to the study of algebraic structure that has become an important tool in theoretical computing science, particularly for semantics-based research. The notion of a limit in category theory generalizes various types of universal constructions that occur in diverse areas of mathematics. In our paper we illustrate how to represent some parts of infinitesimal calculus in categorical structures.
\end{abstract}

\section{Introduction}

Nowadays science and technology are indispensable parts of the global world. Their expansion simplifies the work in many branches and of course the daily life [13]. In day to day life we are often interested in the extent to which a change in one quantity affects a change in another related quantity. This is called a rate of change. Differential calculus, a part of mathematics, is about describing in a precise fashion the ways in which related quantities change. Differential and integral calculus are dual fields and together they form a base for infinitesimal calculus $[3-5,7,11]$. Infinitesimal calculus is a part of mathematics concerned with finding the slope of curves, areas under curves, minima and maxima, and other geometric and analytic problems. On the other hand, the category theory is an area of study in mathematics that examines in abstract way the properties of particular mathematical concepts by formalizing them as collections of objects and arrows (called morphisms, although this term also has a specific, non category-theoretical meaning), where these collections satisfy some basic conditions $[1,2]$. The category 
theory is a branch of mathematics that has been developed over the last fifty years, and it has been concerned with the study of algebraic structures [9]. Many significant areas of mathematics and informatics can be formalized as categories, and the use of the category theory allows many intricate and subtle mathematical results in these fields to be stated, and proved, in a much simpler way than without the use of categories. Functions are mostly represented by morphisms from a domain into a codomain of a function, and we can consider them as the structures enclosable into a category [10]. In our paper we show the rôle of categorical structures in infinitesimal calculus - we construct a diagram of functions and we show how to find a categorical limit of that diagram. In the second part of the article we show how to express derivatives in another way in categories.

\section{Basic notions about the category theory}

A category $\mathbf{C}$ is a mathematical structure consisting of objects, e.g. $A, B, \ldots$ and of morphisms of the form $f: A \rightarrow B$ between them. Every object has the identity morphism $i d_{A}: A \rightarrow A$ and morphisms are composable. Because the objects of a category can be arbitrary structures, categories are useful in computer science $[2,10]$, where we often use more complex structures not expressible by sets. Morphisms between categories are called functors, e.g. a functor $F: \mathbf{C} \rightarrow \mathbf{D}$ from a category $\mathbf{C}$ into a category $\mathbf{D}$ considered as a structure-preserving mapping between categories.

\section{Categorical limit}

The notion of a limit in the category theory generalizes various types of universal constructions that occur in diverse areas of mathematics. It can show very precisely how thematically similar constructions of different types of objects, such as the product of sets or groups of topological spaces, are instances of the same categorical construct [8]. Limits and dually colimits in category $\mathbf{C}$ are defined by means of diagrams in $\mathbf{C}$, and we define the categorical limit as limits of diagrams [9]. In any category $\mathbf{C}$ a diagram $\mathrm{D}$ consists of

- the class of objects $D_{i}$, for $i \in I$, in category $\mathbf{C}$

- the set of morphisms $\mathrm{D}(i, j)$, which is defined for any two indexes $i, j$ as a subset of homset

$$
\mathrm{D}(i, j) \subseteq \operatorname{Hom}\left(D_{i}, D_{j}\right)
$$

For defining the limit of diagram we introduce a notion cone in category. Let $G$ by a graph and $\mathbf{C}$ by a category. Let $\mathrm{D}: G \rightarrow \mathbf{C}$ be a diagram in category $\mathbf{C}$. Cone in $\mathbf{C}$ (in the Fig. 1) consists of 
- the base constructed by diagram $D$;

- apex $W$ together with the family of morphisms $\left\{p_{i} \mid i \in I\right\}$ such that it holds $p_{i}: W \rightarrow D_{i}$.

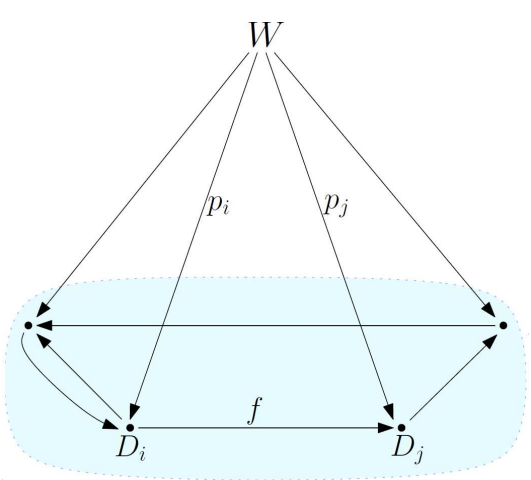

Fig. 1. Commutative cone in category

We usually write a cone as $\left\{p_{i}: W \rightarrow D_{i}\right\}$ or simply $p_{i}: W \rightarrow D_{i}$. A limit of the diagram $\mathrm{D}$ is an object $W$ together with the cone $\left\{p_{i}: W \rightarrow D_{i}\right\}$, for $i \in I$ which has the following universal property:

- for any cone $\left\{q_{i}: X \rightarrow D_{i}\right\}$ there exists exactly one morphism $f: X \rightarrow W$ such that it holds:

$$
f \circ p_{i}=q_{i}
$$

for all $i \in I$. The limit of the diagram $\mathrm{D}$ is depicted in the Figure 2 .

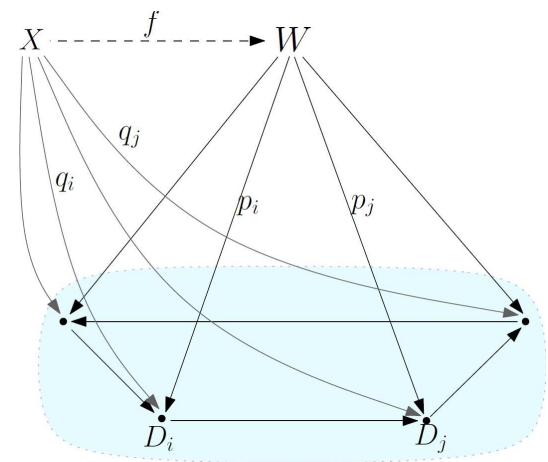

Fig. 2. The limit of the diagram D

The limit of the diagram $\mathrm{D}$ is usually written as

$$
\lim \mathrm{D}=\left\{W \stackrel{p_{i}}{\longrightarrow} D_{i}\right\}
$$

or just $\lim \mathrm{D}=W$. 


\section{Categorical structures in integral calculus}

Assume the category of sets for integral calculus Int, where morphisms are functions between objects. For instance, the cosine function is the following morphism

$$
\cos : R \rightarrow\langle-1 ; 1\rangle
$$

Antiderivative (primitive function) to function cos we denote $p$. It holds

$$
p(x)=\int \cos x d x=\sin x+c .
$$

The value of $p(x)$ depends on the value of constant of integration $c$. Then we can consider the antiderivative as a family of following morphisms

$$
p_{i}: R \rightarrow\left\langle-1+c_{i} ; 1+c_{i}\right\rangle
$$

for $i \in I$.

Let $I=\{1 ; 2 ; 3 ; 4 ; 5 ; 6\}$ be a set of indexes and $C=\left\{c_{1} ; c_{2} ; c_{3} ; c_{4} ; c_{5} ; c_{6}\right\}$ be a set of constants of integration. Let the values of constants be given by the following formula (for $i \in I$ )

$$
c_{i}=i-3 .
$$

We define six antiderivatives to the function cos which differ only in constants of integration (Tab. 1).

Table 1

\begin{tabular}{ccll}
\multicolumn{4}{c}{ Antiderivatives of the cosine function } \\
\hline$i$ & $c_{i}$ & $p_{i}$ & $p_{i}(x)$ \\
\hline $\mathbf{1}$ & -2 & $p_{1}: R \rightarrow\langle-3 ;-1\rangle$ & $p_{1}(x)=\sin x-2$ \\
$\mathbf{2}$ & -1 & $p_{2}: R \rightarrow\langle-2 ; 0\rangle$ & $p_{2}(x)=\sin x-1$ \\
$\mathbf{3}$ & 0 & $p_{3}: R \rightarrow\langle-1 ; 1\rangle$ & $p_{3}(x)=\sin x$ \\
$\mathbf{4}$ & 1 & $p_{4}: R \rightarrow\langle 0 ; 2\rangle$ & $p_{4}(x)=\sin x+1$ \\
$\mathbf{5}$ & 2 & $p_{5}: R \rightarrow\langle 1 ; 3\rangle$ & $p_{5}(x)=\sin x+2$ \\
$\mathbf{6}$ & 3 & $p_{6}: \mathrm{R} \rightarrow\langle 2 ; 4\rangle$ & $p_{6}(\mathrm{x})=\sin x+3$ \\
\hline
\end{tabular}

Between the codomains of particular antiderivatives $p_{i}$ we define functions $v_{i}$ as follows: 


$$
v_{i}(x)= \begin{cases}x+1 & \text { for } i \leq 5 \\ x-5 & \text { for } i=6\end{cases}
$$

Now we can express the functions $v_{i}$ as follows (Tab. 2).

Table 2

Functions in the base of cone

\begin{tabular}{cll}
\hline$i$ & \multicolumn{1}{c}{$v_{i}$} & \multicolumn{1}{c}{$v_{i}(x)$} \\
\hline $\mathbf{1}$ & $v_{1}:\langle-3 ;-1\rangle \rightarrow\langle-2 ; 0\rangle$ & $v_{1}(x)=x+1$ \\
$\mathbf{2}$ & $v_{2}:\langle-2 ; 0\rangle \rightarrow\langle-1 ; 1\rangle$ & $v_{2}(x)=x+1$ \\
$\mathbf{3}$ & $v_{3}:\langle-1 ; 1\rangle \rightarrow\langle 0 ; 2\rangle$ & $v_{3}(x)=x+1$ \\
$\mathbf{4}$ & $v_{4}:\langle 0 ; 2\rangle \rightarrow\langle 1 ; 3\rangle$ & $v_{4}(x)=x+1$ \\
$\mathbf{5}$ & $v_{5}:\langle 1 ; 3\rangle \rightarrow\langle 2 ; 4\rangle$ & $v_{5}(x)=x+1$ \\
$\mathbf{6}$ & $v_{6}:\langle 2 ; 4\rangle \rightarrow\langle-3 ;-1\rangle$ & $v_{6}(x)=x-5$ \\
\hline
\end{tabular}

Next we define the composition of functions $v_{i}$, and for that we formulate the relation between domains and codomains of functions $v_{i}$ :

$$
\operatorname{dom}\left(v_{i}\right)=\left\{\begin{array}{c}
\operatorname{cod}\left(v_{i-1}\right) \text { for } i>1 \\
\operatorname{cod}\left(v_{6}\right) \text { for } i=1
\end{array}\right.
$$

From the functions $v_{i}$ we construct the diagram $\mathrm{D}$ in the Figure 3 which represents the base of a cone.

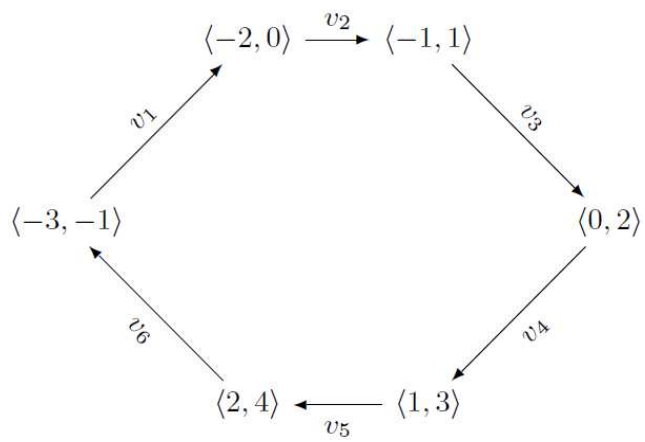

Fig. 3. Diagram of mapping the interval to interval

Then we construct a commutative cone over the diagram in the Figure 3, such as it is depicted in the Figure 4. The existence of a limit of diagram D we show in the following way. Let $X$ be an arbitrary object of the category Int which is the apex of a cone over the diagram D. It means that there exists a family of functions 


$$
q_{i}: X \rightarrow\left\langle-1+c_{i} ; 1+c_{i}\right\rangle
$$

for $i \in I$. It is evident that there exists the only morphism in: $X \rightarrow R$, which is an inclusion. Therefore we can say that the limit of the diagram $\mathrm{D}$ is the set $R$ of reals, $\lim \mathrm{D}=R$.

Limit of the diagram $\mathrm{D}$ is depicted in the Figure 5.

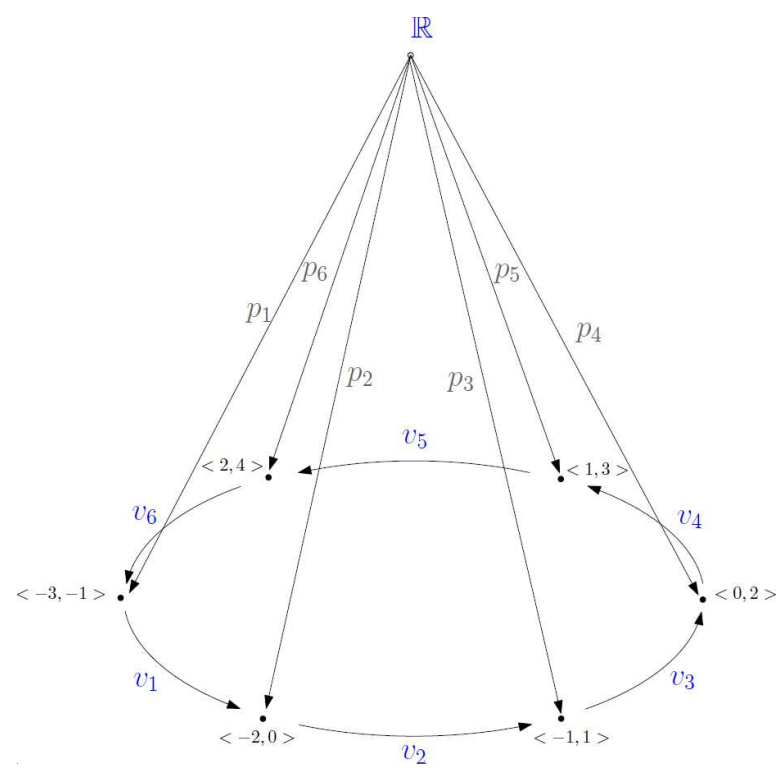

Fig. 4. Commutative cone of antiderivatives

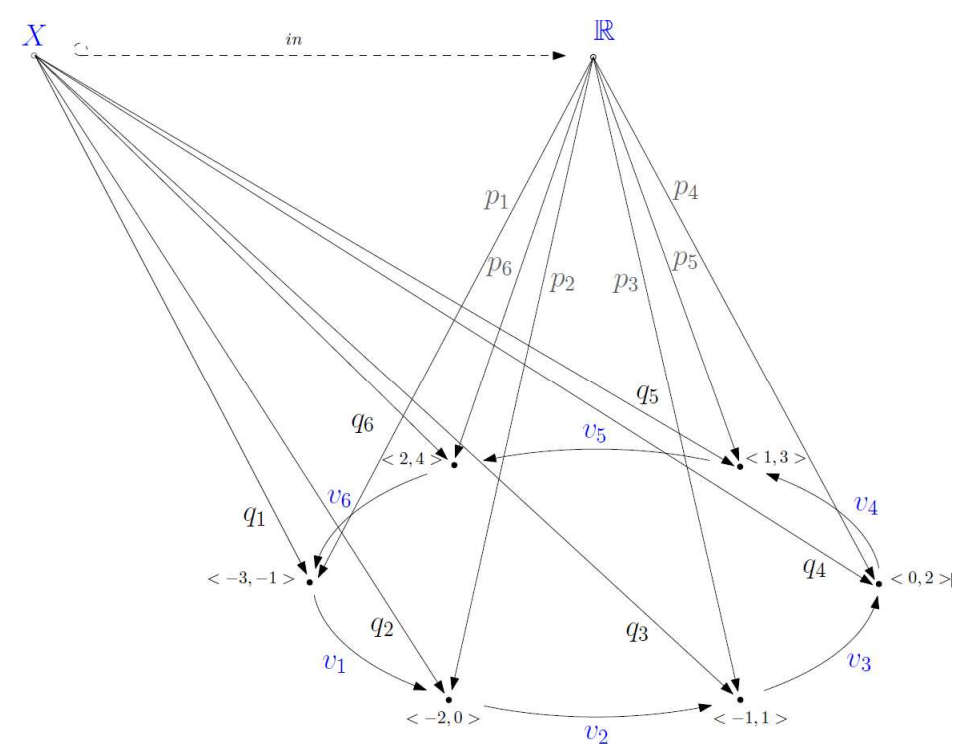

Fig. 5. Commutative cones of antiderivatives with the limit $R$ 


\section{Categorical structures in differential calculus}

The practical applications of differential calculus are very widely ranging. Suffice it to say that differential calculus is an indispensable tool in every branch of science and engineering. In elementary mathematics there are two main applications of differential calculus. One is to help in sketching curves, and the other is in optimisation problems [11].

We could also say that differential calculus is a procedure for finding the exact derivative directly from the formula of a function, without having to use graphical methods. In practise we use a few rules that tell us how to find the derivative of almost any function that we are likely to encounter.

The derivative of a function at a chosen input value describes the best linear approximation of the function near that input value. Informally, the derivative is the ratio of the infinitesimal change of the output over the infinitesimal change of the input producing that output. For a real-valued function of a single real variable, the derivative at a point equals the slope of the tangent line to the graph of the function at that point. The process of finding a derivative is called differentiation. The reverse process is called antidifferentiation. The fundamental theorem of calculus states that antidifferentiation is the same as integration. Differentiation and integration constitute two fundamental operations in single-variable calculus.

\subsection{Derivatives in category}

For defining the derivatives in category we consider the arrow category Der $\rightarrow$ over the category of sets. The arrow category, also called a comma category is defined for each category [6]. Any arrow category $\mathbf{C}^{\rightarrow}$ over the base category $\mathbf{C}$ has as objects all morphisms from the base category. Then morphisms of the arrow category are morphisms defined as tuples between domains and codomains of objects.

Because the derivative $f^{\prime}$ to function $f$ is also a function, we can construct the base category as a category of sets Der, where objects are sets (the domains and codomains of functions) and morphisms are functions. The arrow category Der $\overrightarrow{ }$ over a base category of sets exists according to the definition, so we can define any function as an object in arrow category. Morphisms of $\mathbf{D e r}{ }^{\rightarrow}$ are the operations of differentiation which assign to any function $f$ its derivative $f^{\prime}$ according to the definition.

The category Der $\rightarrow$ is defined as follows:

1. objects are functions $f, g, h, \ldots$ and their derivatives $f, f^{\prime}, \ldots$. The functions are arrows in the base category Der ;

2. morphisms are tuples $(D, C)$ of mappings between domains and codomains of objects: 


$$
(D, C): f \rightarrow f^{\prime},
$$

is a differentiation operation which assigns to function $f$ its derivative $f^{\prime}$, where $D$ is a mapping between domains and $C$ between codomains. The tuple $(D, C)$ we denote as der morphism.

3. for any object $f$ there an identity morphism is defined which sends any object to itself:

$$
i d_{f}: f \rightarrow f .
$$

4. composition of morphisms is defined as follows: for two morphisms $\operatorname{der}_{1}: f \rightarrow f^{\prime}$ and $\operatorname{der}_{2}: f^{\prime} \rightarrow f^{\prime \prime}$, their composition $\operatorname{der}_{2} \circ d e r_{1}: f \rightarrow f^{\prime \prime}$ is a new morphism that assigns to function $f$ it's the second derivative, because the first derivative of $f^{\prime}$ is defined as follows, $\left(f^{\prime}\right)^{\prime}=f^{\prime \prime}$.

5. composition of morphisms is associative operation defined as follows:

$$
\left(d e r_{3} \circ d e r_{2}\right) \circ d e r_{1}=d e r_{3} \circ\left(d e r_{2} \circ d e r_{1}\right) \text {. }
$$

The codomain functor $\operatorname{Cod}:$ Der $^{\rightarrow} \rightarrow$ Der always exists. It assigns to any object $f$ in $\operatorname{Der}^{\rightarrow}$ its codomain $\operatorname{cod}(f)$ in Der, and to any morphism der: $f \rightarrow f^{\prime}$ in Der $^{\rightarrow}$ the arrow between codomains of der in Der (Fig. 6).

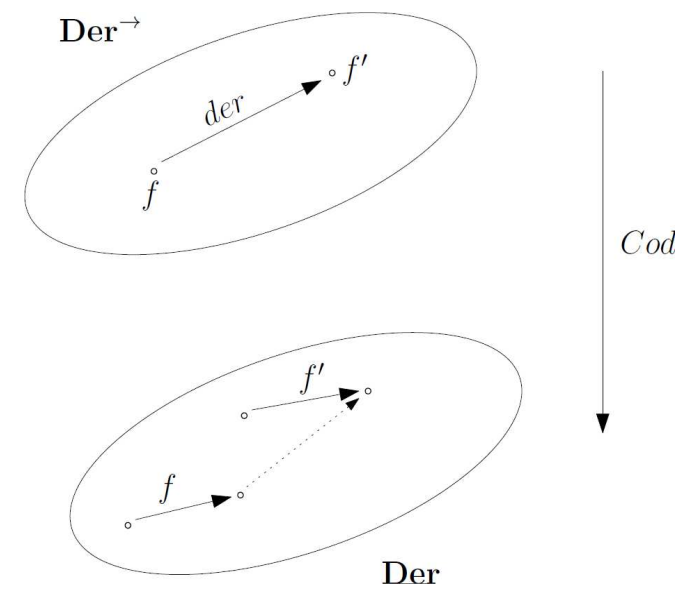

Fig. 6. The codomain functor between categories

\subsection{Example of expressing the derivatives in category}

For instance we take the function of natural logarithm, $f(x)=\ln x$ which has the following specification: 


$$
f:(0 ; \infty) \rightarrow R
$$

The logarithmic function is a morphism with the domain of positive real numbers and the codomain is the set of all real numbers. When finding the derivative of function, we use rules for derivation. For a logarithmic function its derivative is as follows:

$$
f^{\prime}(x)=(\ln x)^{\prime}=\frac{1}{x},
$$

which is a well-known basic reciprocal linear rational function, simply called a reciprocal function. This function is defined on the set of non-zero reals, and it sends every real number to its reciprocal value, i.e. its multiplication inverse. The reciprocal function $f^{\prime}(x)=\frac{1}{x}$ has the specification

$$
f^{\prime}: R|\{0\} \rightarrow R|\{0\}
$$

where both the domain and the codomain are both the same - the set of nonzero real numbers. The second derivative of $f(x)=\ln x$ is the first derivative of reciprocal function $f^{\prime}(x)=\frac{1}{x}$ and it is the function

$$
f^{\prime \prime}(x)=-\frac{1}{x^{2}}
$$

which has the specification

$$
f^{\prime \prime}: \mathrm{R} \backslash\{0\} \rightarrow(-\infty ; 0) \text {. }
$$

All functions listed above are depicted in the Figure 7.

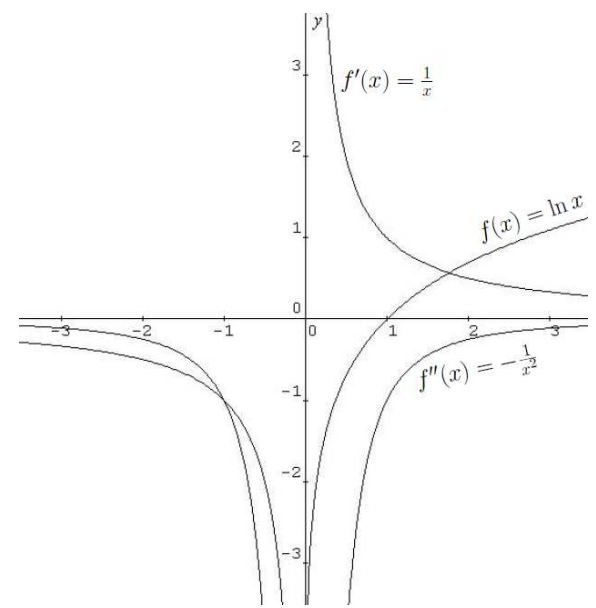

Fig. 7. Natural logarithm function and its the first and second derivative 
It holds that second derivative of $\ln x$ is a function $f^{\prime \prime}(x)=-\frac{1}{x^{2}}$ which is the first derivative of function $f^{\prime}(x)=\frac{1}{x}$,

$$
f^{\prime \prime}(x)=\left(f^{\prime}(x)\right)^{\prime}
$$

In the category Der, the functions $f, f^{\prime}$ and $f^{\prime \prime}$ are the following morphisms in category:

$$
\begin{array}{lll}
f:(0 ; \infty) \rightarrow R & \operatorname{dom}(f)=(0 ; \infty) & \operatorname{cod}(f)=R \\
f^{\prime}: R \backslash\{0\} \rightarrow R \backslash\{0\} & \operatorname{dom}\left(f^{\prime}\right)=R \backslash\{0\} & \operatorname{cod}\left(f^{\prime}\right)=R \backslash\{0\} \\
f^{\prime \prime}: R \backslash\{0\} \rightarrow(-\infty ; 0) & \operatorname{dom}\left(f^{\prime \prime}\right)=R \backslash\{0\} & \operatorname{cod}(f)=(-\infty ; 0)
\end{array}
$$

and the sets $(0 ; \infty), R, R \backslash\{0\}$ and $(-\infty ; 0)$ are objects in this category. For any object $A$ the identity morphism is defined as follows:

$$
i d_{A}: A \rightarrow A,
$$

and morphisms are composable according to the obvious rules for composition of functions.

In category $\operatorname{Der}^{\rightarrow}$ the functions $f, f^{\prime}$ and $f^{\prime \prime}$ are objects in this category. The morphisms between objects are differentations, denoted der :

$$
\text { der }: f \rightarrow f^{\prime},
$$

and they are defined as tuples of two arrows for mapping of domains and codomains of objects, $\operatorname{der}=(D, C)$, where

$$
\begin{aligned}
& D: \operatorname{dom}(f) \rightarrow \operatorname{dom}\left(f^{\prime}\right), \\
& C: \operatorname{cod}(f) \rightarrow \operatorname{cod}\left(f^{\prime}\right) .
\end{aligned}
$$

The identity is defined for each object,

$$
i d_{f}: f \rightarrow f,
$$

is the zero derivation which is an identity mapping: it sends any function to itself. The composition of functions represents the gradual increasing of order of differentiation. Here, for the functions $f^{\prime}(x)=\frac{1}{x}$ and $f^{\prime \prime}(x)=-\frac{1}{x^{2}}$, the differentiations are:

- $d e r_{1}: f \rightarrow f^{\prime}$ for the first derivative of $f$;

- $d e r_{2}: f^{\prime} \rightarrow f^{\prime \prime}$ for the first derivative of $f^{\prime}$; 
By composition of $d e r_{1}$ and $d e r_{2}$ we obtain a new morphism $d e r_{2} \circ d e r_{1}$ (we can denote it also $d e r_{12}$ ):

$$
\operatorname{der}_{2} \circ d e r_{1}: f \rightarrow f^{\prime \prime}
$$

and it is the second derivative of $f$ :

$$
f^{\prime \prime}(x)=(\ln x)^{\prime \prime}=-\frac{1}{x^{2}} .
$$

For the composition it holds the following commutative diagram (Fig. 8).

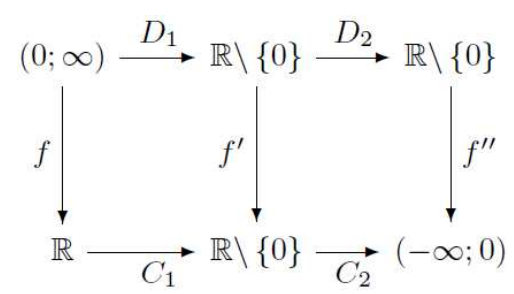

Fig. 8. Diagram of second derivative

From the diagram in Figure 8 the following equality holds:

$$
C_{2} \circ C_{1} \circ f=f^{\prime \prime} \circ D_{2} \circ D_{1} .
$$

The relation between categories Der and Der $^{\rightarrow}$ is expressed by the codomain functor

$$
\text { Cod }: \text { Der } \rightarrow \text { Der }
$$

This functor assigns to every object is $\mathbf{D e r}^{\rightarrow}$ its codomain - a set in Der :

$$
\operatorname{Cod}(f)=B
$$

for any $f: A \rightarrow B$, and any morphism in $\mathbf{D e r}^{\rightarrow}$ it sends to a morphism between the appropriate codomains:

$$
\operatorname{Cod}(\operatorname{der})=C
$$

for any $\operatorname{der}: f \rightarrow f^{\prime}$, where morphism $C$ is

$$
C: \operatorname{cod}(f) \rightarrow \operatorname{cod}\left(f^{\prime}\right) \text {. }
$$




\section{Conclusion}

In this paper we have shown the construction of diagram of antiderivatives in categories. We constructed a commutative cone of antiderivatives to the cosine function and we defined family of functions for the base of commutative cone of functions. Finally, we found a limit of the diagram of functions. The second part of the article follows our approach for derivatives. The aim of our paper was an illustration of usability of categories in various fields of mathematics. Our next goal is to investigate how to implement mathematical expressions of some parts of infinitesimal calculus in practical approach [12].

\section{Acknowledgment}

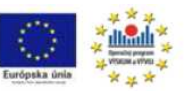

This work is the result of the project implementation: Center of Information and Communication Technologies for Knowledge Systems (ITMS project code: 26220120030) supported by the Research \& Development

Operational Program funded by the ERDF.

\section{References}

[1] Hrušková R., Social skills in the context of science and technology, [in:] Proceedings of scientific works - Veda a technika v procese globalizácie a humanizácia vzdelávania na technických univerzitách, Slovak University of Technology in Bratislava, 2009 (in Slovak).

[2] Bourbaki N., Integration I, Springer Verlag 2004.

[3] Burton D.M., The History of Mathematics: An Introduction (6th ed.), McGraw- Hill 2005.

[4] Forster O., Analysis 1. Differential- und Integralrechnung einer Veränderlichen. 7. Au. ViewegVerlag, 2004.

[5] Keisler H.J., Elementary Calculus: An Infinitesimal Approach, On-line Edition. Copyright 2000/revised 2012, University of Wisconsin, http://www.math.wisc.edu/ keisler/calc.html

[6] Thomas C., Introduction to Differential Calculus, University of Sydney 1997.

[7] Awodey S., Category Theory, Carnegie Mellon University 2005.

[8] Barr M., Wells C., Category Theory for Computing Science, Prentice Hall International, 1990, ISBN 0-13-120486-6.

[9] Novitzká V., Slodičák V., Categorical structures and their application in informatics, Equilibria, Košice 2010, in Slovak.

[10] Slodičák V., Macko P., New approaches in functional programming using algebras and coalgebras, European Joint Conferences on Theory and Practice of Software-ETAPS 2011, Universität des Saarlandes, Saarbrücken, Germany, 13-23, ISBN 978-963-284-188-5.

[11] Messick S., Limits in category theory, University of Chicago, USA 2007.

[12] Jacobs B., Categorical Logic and Type Theory. No. 141 in Studies in Logic and the Foundations of Mathematics. North Holland, Amsterdam 1999.

[13] Luković I., Ristić S., Popović A., Mogin P., An approach to the platform independent specification of a business application, Proceedings of the $23^{\text {rd }}$ Central European Conference on Information and Intelligent Systems - CECIIS'2012, 19 ${ }^{\text {th }}-21^{\text {st }}$ Sept 2012, University of Zagreb, Varaždin, Croatia, 449-456. 
Zusammenfassung. Die Theorie von Kategorien ist der Bereich von Mathematik und sie dient vor allem für das Studium der algebraischen Strukturen. Sie wird aber sehr oft auch in Informatik geltend gemacht. Manche bedeutende mathematische Bereiche kann man mithilfe der Kategorien darstellen und das ermöglicht mit den mathematischen Strukturen viel einfacher zu arbeiten als ohne Anwendung der Kategorien. Der Grund der Infinitesimalrechnung bilden zwei duale Bereiche - Differential- und Integralrechnung. In unserem Beitrag orientieren wir uns auf die Konstruktion des Diagramms von Stammfunktionen zur Funktion Kosinus. Von diesen Funktionen konstruieren wir den kommutativen Kegel und wir finden seinen Grenzwert. In dem zweiten Teil des Artikels zeigen wir den Ausdruck der Derivationen von Funktionen in der Kommakategorie und wir konstruieren den kodomänen Funktor zwischen der Kategorie der Derivationen und der Kategorie der Mengen für differenzierbare Funktionen. 